\title{
Numerical Simulation of Inviscid Compressible Fluid Flow Around Moving Bodies
}

\section{Jitka Klečková}

Research and Testing Institute Pilsen s.r.o., Tylova 1581/46, 30100 Plzeň. Czech Republic. E-mail: kleckova@vzuplzen.cz

The article presents numerical code which was developed for solution of inviscid compressible fluid flow in domains with deforming boundaries. This computational method for the numerical solution of the non-linear system of Euler equations in time-dependent domains was designed as the first step of solution of fluid-structure interaction problem. Arbitrary Lagrangian - Eulerian (ALE) description of continuum, combining Eulerian and Lagrangian approach, was used to describe the inviscid fluid flow in time-dependent domain. The spatial discretization was provided by finite volume method adapted for triangular computational grids. Inviscid fluxes were discretized by the Rusanov flux scheme and Van Leer flux splitting scheme. The computational code was validated using a case of inviscid fluid flow around vibrating airfoil NACA 0012 which was experimentally investigated by AGARD group in 1982. Boundary conditions and simulation parameters were set according to the conditions of experimental measurement and the rotation angle of the body was defined by a time-dependent function. The numerical results are compared with experimental data and results of other authors. The algorithm for the mesh deformation is presented.

Keywords: Inviscid fluid, ALE form of Euler equations, deforming mesh, NACA 0012

\section{Acknowledgement}

I would like to gratefully acknowledge the support of the research project TA ČR $n$. TE01020068 "Centre of research and experimental development of reliable energy production".

\section{References}

[1] FEISTAUER, M., KUČERA, V., PROKOPOVÁ, J. (2010). Discontinuous Galerkin solution of compressible flow in time-dependent domains. Mathematics and Computers in simulation, Vol. 80, Issue 8, Elsevier, p. 1612-1623.

[2] PERSSON, P., O., BONET, J., PERAIRE, J. (2009). Discontinuous Galerkin solution of the Navier - Stokes equations on deformable domains. Computer Methods in Applied Mechanics and Engineering, Vol. 198, Issue 1720, Elsevier, p. 1585-1595.

[3] BUBLÍK, O., VIMMR, J., JONÁŠOVÁ, A. (2015). Comparison of discontinuous Galerkin time integration schemes for the solution of flow problems with deformable domains. Applied Mathematics and Computation, Elsevier, (accepted for publication).

[4] LANDON, R., H. (1982). NACA 0012. Oscillatory and transient pitching. Compendium of unsteady aerodynamics measurements, AGARD Report 702, p. 3-1 - 3-25.

[5] FURMÁNEK, P., FÜRST, J., KOZEL, K. (2010). ALE method for unsteady flow computations. ECCOMAS CFD 2010. CD-ROM, ECCOMAS, Lisabon. 